\title{
Minimum distance of Orthogonal Line-Grassmann Codes in even characteristic
}

\author{
Ilaria Cardinali and Luca Giuzzi
}

\begin{abstract}
In this paper we determine the minimum distance of orthogonal line-Grassmann codes for $q$ even. The case $q$ odd was solved in 3. For $n \neq 3$ we also determine the second smallest distance. Furthermore, we show that for $q$ even all minimum weight codewords are equivalent and that symplectic line-Grassmann codes are proper subcodes of codimension $2 n$ of the orthogonal ones.
\end{abstract}

Keywords: Orthogonal Grassmannian, Projective Code, Minimum Distance MSC: 51A50, 51E22, 51A45

\section{Introduction}

A projective code $\mathcal{C}(\Omega)$ is an error correcting code determined by a projective system, that is a set $\Omega$ of $N$ distinct points of a finite projective space. More in detail, $\mathcal{C}(\Omega)$ is a linear code of length $N$ generated by the rows of a matrix $G$ whose columns are the coordinates of the points of $\Omega$ with respect to some fixed reference system. In general, $\mathcal{C}(\Omega)$ is not uniquely determined by $\Omega$, but it turns out to be unique up to monomial equivalence; as such its metric properties with respect to Hamming's distance depend only on the set of points under consideration. With a slight abuse of notation, which is however customary when dealing with projective codes, we shall speak of $\mathcal{C}(\Omega)$ as the code defined by $\Omega=\left\{\omega_{1}, \omega_{2}, \ldots, \omega_{N}\right\}$ where the $\omega_{i}$ s are fixed vector representations of the points of the projective system.

As mentioned above, the parameters $\left[N, K, d_{\min }\right]$ of $\mathcal{C}(\Omega)$ depend only on the pointset $\Omega$; in particular, the length $N$ is the size of $\Omega$ and the dimension $K$ is the (vector) dimension of the subspace of $W$ spanned by $\Omega$. It is straightforward to show that the minimum distance $d_{\min }$ is

$$
d_{\min }=N-\max _{\Pi}|\Omega \cap \Pi|,
$$

as $\Pi$ ranges among all hyperplanes of the space $\mathrm{PG}(\langle\Omega\rangle)$; we refer to 13 for further details.

The codes associated with polar $k$-Grassmannians of either orthogonal, symplectic or Hermitian type have been introduced respectively in [1], [4] and [5]. In the case of line-Grassmannians, that is for $k=2$, the following results are known: in the symplectic case it has been shown in [4] that the minimum distance is $q^{4 n-5}-q^{2 n-3}$ for any $q$; in the orthogonal case it has been shown in [1, Main Result 2] that the minimum distance is $d_{\min }=q^{3}-q^{2}$ for $n=2$ for any $q$ and in [3] that the minimum distance is $d_{\min }=q^{4 n-5}-q^{3 n-4}$ for $q$ odd. Similar results hold in the Hermitian case, see [5].

The aim of the present paper is to determine the minimum distance of orthogonal lineGrassmann codes for $q$ even. We are also able to determine the second smallest distance in almost all cases. Our main result is the following. 
Main Theorem. For $q$ even, the minimum distance of a line orthogonal Grassmann code is

$$
d_{\min }=q^{4 n-5}-q^{3 n-4}
$$

and all words of minimum weight are projectively equivalent. Furthermore, the second smallest distance for $n \neq 3$ is $q^{4 n-5}-q^{2 n-3}$.

Using the aforementioned results of [1, 3] this leads to the following general result.

Corollary 1.1. The parameters $\left[N, K, d_{\mathrm{min}}\right]$ of a line orthogonal Grassmann code are

$$
N=\frac{\left(q^{2 n}-1\right)\left(q^{2 n-2}-1\right)}{(q-1)\left(q^{2}-1\right)}, \quad K=\left\{\begin{array}{ll}
(2 n+1) n & q \text { odd } \\
(2 n+1) n-1 & q \text { even },
\end{array} \quad d_{\min }=q^{4 n-5}-q^{3 n-4} .\right.
$$

Note that for $q$ odd and $n=2$, by [3, Corollary 3.8], the minimum weight codewords lie on two orbits under the action of the linear automorphism group of the code.

The structure of the paper is as follows. In Section 2 we set the notation and introduce some preliminary results. In particular, in Section 2.1] we recall some basic results on polar Grassmannians and their associated codes and in Section 2.2 we describe in detail a fundamental formula for the computation of weights of codewords in a projective code. In Section 3 we shall prove our Main Theorem.

For further details on the actual construction of orthogonal and symplectic line-Grassmann codes, we refer to [2] where some efficient algorithms for encoding, decoding and error-correction have been presented.

\section{Preliminaries}

\subsection{Grassmann and Polar Grassmann codes}

Let $V:=V(2 n+1, q)$ be a vector space of odd dimension defined over a finite field $\mathbb{F}_{q}$ of order $q$ and denote by $\mathcal{G}_{k}$ the Grassmannian of the $k$-subspaces of $V$.

For any $k<\operatorname{dim}(V)$, let $\varepsilon_{k}: \mathcal{G}_{k} \rightarrow \operatorname{PG}\left(\bigwedge^{k} V\right)$ be the usual Plücker embedding, mapping a point $\left\langle v_{1}, \ldots, v_{k}\right\rangle$ of $\mathcal{G}_{k}$ to the projective point $\left\langle v_{1} \wedge \cdots \wedge v_{k}\right\rangle$ of $\operatorname{PG}\left(\bigwedge^{k} V\right)$ :

$$
\varepsilon_{k}:\left\langle v_{1}, \ldots, v_{k}\right\rangle \mapsto\left\langle v_{1} \wedge \cdots \wedge v_{k}\right\rangle .
$$

Let $\eta: V \rightarrow \mathbb{F}_{q}$ be a fixed non-degenerate quadratic form over $V$ and denote by $\Delta_{k}$ the orthogonal Grassmannian associated to $\eta$, that is $\Delta_{k}$ is the geometry whose points are the $k^{-}$ subspaces of $V$ which are totally singular for $\eta$ and whose lines are defined as follows

- if $k<n$, then $\ell_{X, Y}:=\{Z: X<Z<Y: \operatorname{dim} Z=k\}$, with $\operatorname{dim} X=k-1, \operatorname{dim} Y=k+1$ and $Y$ totally singular;

- if $k=n$, then $\ell_{X}:=\left\{Z: X<Z<X^{\perp_{\eta}}\right.$ : $\left.\operatorname{dim} Z=n\right\}$, with $\operatorname{dim} X=n-1, Z$ totally singular and $X^{\perp_{\eta}}:=\{y \in V: \beta(x, y)=0$ for all $x \in X\}$, where $\beta$ is the sesquilinearization of $\eta$.

For $k<n, \Delta_{k}$ is a proper subgeometry of $\mathcal{G}_{k}$. In any case, for $k \leq n$ the point-set of $\Delta_{k}$ is always a subset of that of $\mathcal{G}_{k}$.

Put $\varepsilon_{k}\left(\mathcal{G}_{k}\right):=\left\{\varepsilon_{k}(X): X\right.$ is a point of $\left.\mathcal{G}_{k}\right\}$ and $\varepsilon_{k}\left(\Delta_{k}\right)=\left\{\varepsilon_{k}(Y): Y\right.$ is a point of $\left.\Delta_{k}\right\}$. Then, the above statement reads as $\varepsilon_{k}\left(\Delta_{k}\right) \subseteq \varepsilon_{k}\left(\mathcal{G}_{k}\right) \subseteq \mathrm{PG}\left(\bigwedge^{k} V\right)$.

We warn the reader that throughout the paper we will consider vectors and vector dimensions but we will adopt projective terminology. 
Theorem $2.1(\underline{6})$. Let $\varepsilon_{k}: \Delta_{k} \rightarrow \operatorname{PG}\left(\bigwedge^{k} V\right)$ be the restriction of the Plücker embedding to the orthogonal Grassmannian $\Delta_{k}$ and let $W_{k}:=\left\langle\varepsilon_{k}\left(\Delta_{k}\right)\right\rangle$. Then,

$$
\operatorname{dim} W_{k}= \begin{cases}\left(\begin{array}{c}
2 n+1 \\
k
\end{array}\right) & \text { if } q \text { is odd } \\
\left(\begin{array}{c}
2 n+1 \\
k
\end{array}\right)-\left(\begin{array}{c}
2 n+1 \\
k-2
\end{array}\right) & \text { if } q \text { is even }\end{cases}
$$

For more information on embeddings of orthogonal line-Grassmannians we refer to [7].

The image $\varepsilon_{k}\left(\mathcal{G}_{k}\right)$ is a projective system in $\operatorname{PG}\left(\bigwedge^{k} V\right)$ and the projective code $\mathcal{C}\left(\mathcal{G}_{k}\right)$ is called $k$-Grassmann code. Grassmann codes have been introduced in [11, 12] as generalizations of first order Reed-Muller codes and they have been extensively investigated ever since; see [8, 9, 10, 11, 12. Their parameters, as well as some of their higher weights, have been fully determined in [10].

The set $\Omega:=\varepsilon_{k}\left(\Delta_{k}\right)$ is a projective system of $\mathrm{PG}\left(W_{k}\right) \subseteq \mathrm{PG}\left(\bigwedge^{k} V\right)$, hence it is natural to consider the projective code $\mathcal{P}_{n, k}:=\mathcal{C}(\Omega)$ arising from $\Omega$. The codes $\mathcal{P}_{n, k}:=\mathcal{C}(\Omega)$ are called orthogonal $k$-Grassmann codes and they were introduced in [1. Theorem 2.1 immediately provides the length $N$ as the number of points of $\Delta_{k}$ and the dimension $K=\operatorname{dim} W_{k}$ of $\mathcal{P}_{n, k}$. A more difficult task is to determine the minimum distance of an orthogonal Grassmann code. In [1] we obtained the exact value of $d_{\min }$ for $n=k=2$ and $n=k=3$; more recently, in [3], it has been shown that for $q$ odd and $k=2$ the minimum distance of $\mathcal{P}_{n, 2}$ is $q^{4 n-5}-q^{3 n-4}$.

We now present in detail a geometric setting in which it is possible to study the weights of a projective code arising from the image under the Plücker embedding $\varepsilon_{k}$ of an arbitrary set of $k$-subspaces.

For any vector space $U$, denote by $U^{*}$ its dual. It is well known that $\left(\bigwedge^{k} V\right)^{*} \cong \bigwedge^{k} V^{*}$. Suppose $\Omega=\left\{\omega_{1}, \ldots, \omega_{N}\right\} \subseteq \varepsilon_{k}\left(\mathcal{G}_{k}\right)$ to be a projective system of $\bigwedge^{k} V$ and take $W:=\langle\Omega\rangle$. Let now

$$
\mathcal{N}(\Omega):=\left\{\varphi \in \bigwedge^{k} V^{*}:\left.\varphi\right|_{\Omega} \equiv 0\right\}
$$

be the annihilator of the set $\Omega$; clearly $\mathcal{N}(\Omega)=\mathcal{N}(W)$. There exists a correspondence between the elements of $\left(\bigwedge^{k} V^{*}\right) / \mathcal{N}(\Omega) \cong W^{*}$ and the codewords of $\mathcal{C}(\Omega)$. More precisely, given any $\varphi \in W^{*}$, the codeword $c_{\varphi}$ corresponding to $\varphi$ is defined as

$$
c_{\varphi}:=\left(\varphi\left(\omega_{1}\right), \ldots, \varphi\left(\omega_{N}\right)\right) .
$$

As $\Omega$ spans $W$ it is immediate to see that $c_{\varphi}=c_{\psi}$ if and only if $\varphi-\psi \in \mathcal{N}(\Omega)$, that is $\varphi=\psi$ as elements of $W^{*}$.

Define the weight $\operatorname{wt}(\varphi)$ of $\varphi$ to be the weight of $c_{\varphi}$, that is

$$
\operatorname{wt}(\varphi):=\operatorname{wt}\left(c_{\varphi}\right)=|\{\omega \in \Omega: \varphi(\omega) \neq 0\}| .
$$

It is well known that linear functionals in $\bigwedge^{k} V^{*}$ are equivalent to $k$-linear alternating forms defined on $V$. In particular, given $\varphi \in \bigwedge^{k} V^{*}$ we can define $\varphi^{*}: V^{k} \rightarrow \mathbb{F}_{q}$ as

$$
\varphi^{*}\left(v_{1}, \ldots, v_{k}\right):=\varphi\left(v_{1} \wedge v_{2} \wedge \cdots \wedge v_{k}\right)
$$

which is a $k$-linear alternating form. Conversely, given a $k$-linear alternating form $\varphi^{*}: V^{k} \rightarrow \mathbb{F}_{q}$, there is a unique element $\varphi \in \bigwedge^{k} V^{*}$ such that

$$
\varphi\left(v_{1} \wedge \ldots \wedge v_{k}\right):=\varphi^{*}\left(v_{1}, \ldots, v_{k}\right)
$$

for any $v_{1}, \ldots, v_{k} \in V$. In particular, $\varphi(u)=0$ for $u=\left\langle v_{1} \wedge v_{2} \wedge \cdots \wedge v_{k}\right\rangle \in \Omega$ if and only if all the $k$-tuples of elements of the vector space $U:=\left\langle v_{1}, \ldots, v_{k}\right\rangle$ are killed by $\varphi^{*}$. With a slight 
abuse of notation, in the remainder of this paper we shall use the same symbol $\varphi$ for both the linear functional and the related $k$-alternating form.

For linear codes the minimum distance is the minimum of the weights of the non-zero codewords; so, in order to obtain the minimum distance of the codes $\mathcal{P}_{n, k}$ we need to determine the maximum number of $k$-spaces of $V$ with are both totally $\eta$-singular and $\varphi$-totally isotropic, where $\varphi$ is an arbitrary $k$-linear alternating form which is not identically null on the elements of $\Delta_{k}$.

\subsection{A recursive formula for the weights}

Take $\varphi \in\left(\bigwedge^{k} V\right)^{*}$ and let $u \in V$. Then, we can define a functional $\varphi_{u} \in\left(\bigwedge^{k-1} V\right)^{*}$ by

$$
\varphi_{u}:\left\{\begin{array}{l}
\bigwedge^{k-1} V \rightarrow \mathbb{F}_{q} \\
x \mapsto \varphi(u \wedge x) .
\end{array}\right.
$$

Define $u \bigwedge^{k-2} V:=\left\{u \wedge y: y \in \bigwedge^{k-2} V\right\} \subseteq \bigwedge^{k-1} V$ and $V_{u}:=V /\langle u\rangle$. Observe that for any $y \in u \bigwedge^{k-2} V$ we have $\varphi_{u}(y)=0$. Since $\bigwedge^{k-1} V_{u}=\left(\bigwedge^{k-1} V\right) /\left(u \bigwedge^{k-2} V\right)$, for $x \in \bigwedge^{k-1} V$, we can define a functional $\widehat{\varphi}_{u} \in\left(\bigwedge^{k-1} V_{u}\right)^{*}$ by

$$
\widehat{\varphi}_{u}:\left\{\begin{array}{l}
\bigwedge^{k-1} V_{u} \rightarrow \mathbb{F}_{q} \\
x+\left(u \bigwedge^{k-2} V\right) \mapsto \varphi_{u}(x) .
\end{array}\right.
$$

Let now $\mathcal{T}$ be a set of $k$-subspaces of $V$ and denote by $\mathbb{T} \subseteq V$ the set of vectors of $V$ belonging to at least one element of $\mathcal{T}$. Put $\varepsilon_{k}(\mathcal{T})=\left\{\varepsilon_{k}(X): X \in \mathcal{T}\right\}$. Define

$$
\mathcal{T}_{u}:=\{X /\langle u\rangle: X \in \mathcal{T}, u \in X\} \subseteq V_{u} \text { and } S_{u}:=\left\langle\mathcal{T}_{u}\right\rangle
$$

Apply the Plücker embedding $\varepsilon_{k-1}: \mathcal{G}_{k-1} \rightarrow \operatorname{PG}\left(\bigwedge^{k-1} V_{u}\right)$ to the elements $X /\langle u\rangle \in \mathcal{T}_{u}$. Put $\Omega_{u}:=\left\{\varepsilon_{k-1}(X /\langle u\rangle): X /\langle u\rangle \in \mathcal{T}_{u}\right\}$. The set $\Omega_{u}$ can be regarded as a projective system of $\bigwedge^{k-1} S_{u}$. We can consider the linear functional $\widetilde{\varphi}_{u}:=\left.\widehat{\varphi}_{u}\right|_{\bigwedge^{k-1} S_{u}}$ which is the restriction of $\widehat{\varphi}_{u}$ to $\bigwedge^{k-1} S_{u}$. As recalled in Section 2.1, to each codeword of $\mathcal{C}\left(\Omega_{u}\right)$ there correspond exactly one functional $\widetilde{\varphi}_{u}^{\prime} \in\left(\bigwedge^{k-1} S_{u}^{*}\right) / \widetilde{\mathcal{N}}\left(\Omega_{u}\right)$ where

$$
\tilde{\mathcal{N}}\left(\Omega_{u}\right):=\left\{\psi \in \bigwedge^{k-1} S_{u}^{*}:\left.\psi\right|_{\Omega_{u}} \equiv 0\right\} .
$$

Observe that, given $\widetilde{\varphi}_{u}^{\prime} \in\left(\bigwedge^{k-1} S_{u}^{*}\right) / \widetilde{\mathcal{N}}\left(\Omega_{u}\right)$, there exists a functional $\widetilde{\varphi}_{u} \in\left(\bigwedge^{k-1} S_{u}^{*}\right)$ such that $\operatorname{wt}\left(\widetilde{\varphi}_{u}\right)=\operatorname{wt}\left(\widetilde{\varphi}_{u}^{\prime}\right)$.

Under the set-up introduced above, the following formula holds (see [3, Lemma 2.2]):

$$
\operatorname{wt}(\varphi)=\frac{1}{q^{k}-1} \sum_{u \in \mathbb{T}} \operatorname{wt}\left(\widetilde{\varphi}_{u}\right) .
$$

Note that when $\mathcal{T}$ is the set of all $k$-subspaces of $V$, namely $\mathcal{T}$ is $\mathcal{G}_{k}$, then $\mathbb{T}$ is the pointset of $V$, hence $S_{u}=V_{u}, \bigwedge^{k-1}\left(S_{u} /\langle u\rangle\right)=\bigwedge^{k-1} V_{u}$ and $\widetilde{\varphi}_{u}=\widehat{\varphi}_{u}=\widetilde{\varphi}_{u}^{\prime}$.

Observe also that if $\Omega_{u}$ spans $\bigwedge^{k-1} S_{u}$, then $\tilde{\mathcal{N}}\left(\Omega_{u}\right)$ is trivial and $\widetilde{\varphi}_{u}^{\prime}=\widetilde{\varphi}_{u}$. This happens, for example, in the case of orthogonal Grassmann codes for $q$ odd or $k=2$. Indeed, if we specialize to the case of line orthogonal Grassmann codes (i.e. $k=2$ and $\mathcal{T}=\Delta_{2}$ ), we have that $\mathbb{T}$ is the pointset of the non-degenerate parabolic quadric $\mathcal{Q} \cong Q(2 n, q)$ defined by the quadratic form $\eta$. 
So $\mathcal{T}_{u}$, with $u \in \mathcal{Q}$, is isomorphic to the non-degenerate parabolic quadric $\mathcal{Q}_{u}$ having as points, the lines of $\mathcal{Q}$ through $u$. In this case $\mathcal{T}_{u} \cong \mathcal{Q}_{u}$ is naturally embedded (by $\varepsilon_{1}$ ) as a non-degenerate parabolic quadric $\Omega_{u} \cong Q(2 n-2, q)$ in a $(2 n-1)$-dimensional vector space $S_{u}$. Hence $\Omega_{u}$ spans $S_{u}$ and $\tilde{\mathcal{N}}\left(\Omega_{u}\right)=\{0\}$.

\section{Proof of the Main Theorem}

If $q=2$ and $n=2$, [1, Main Result 2] shows that the minimum distance of the code is $4=2^{3}-2^{2}$. A direct computation proves that all 45 words of minimum weight lie in the same orbit under the action of the automorphism group of the code, which is isomorphic to the orthogonal linear group $G O(5,2)$ in its natural action on $\bigwedge^{2} V$.

Henceforth, we shall assume $q$ to be even and $(q, n) \neq(2,2)$. As mentioned in the Introduction, the case $q$ even and $n=2$ is also covered by [1, Main result 2].

As $\operatorname{dim} V$ is odd, all non-degenerate quadratic forms on $V$ are projectively equivalent. So, for the purposes of the present paper, we can assume to have fixed a basis $B:=\left(e_{1}, \ldots, e_{2 n+1}\right)$ of $V$ such that $\eta$ is

$$
\eta(x):=\sum_{i=1}^{n} x_{2 i-1} x_{2 i}+x_{2 n+1}^{2},
$$

where $\left(x_{i}\right)_{i=1}^{2 n+1}$ are the coordinates of a vector $x \in V$ with respect to $B$.

Let $\beta(x, y):=\eta(x+y)-\eta(x)-\eta(y)$ be the bilinear form associated with $\eta$ by sesquilinearization. As $q$ is even, the bilinear form $\beta$ is degenerate with 1-dimensional radical $N=\{x \in$ $V: \beta(x, y)=0$ for any $y \in V\}=\left\langle e_{2 n+1}\right\rangle$.

The set of totally singular vectors for $\eta$ determine a parabolic quadric $\mathcal{Q} \cong Q(2 n, q)$ in $\operatorname{PG}(V)$. We recall that when $p$ and $q$ are distinct points of $\mathcal{Q}$, the line spanned by $p$ and $q$ is totally singular if and only if

$$
\eta(p)=\eta(q)=\beta(p, q)=0
$$

For any $p \in \mathcal{Q}$, define

$$
p^{\perp \mathcal{Q}}:=\langle u \in \mathcal{Q}:\langle p, u\rangle \subseteq \mathcal{Q}\rangle .
$$

So $p^{\perp \mathcal{Q}}$ is the tangent hyperplane at $p$ to $\mathcal{Q}$. By construction, we also have $p^{\perp \mathcal{Q}}=\{u \in$ $V: \beta(p, u)=0\}$; thus $N \subseteq p^{\perp \mathcal{Q}}$ for any $p \in \mathcal{Q}$ and any line through $N$ is tangent to $\mathcal{Q}$. The point $N$ is called the nucleus of the quadric $\mathcal{Q}$.

By Theorem 2.1, $\Sigma:=\operatorname{PG}\left(\left\langle\varepsilon_{2}\left(\Delta_{2}\right)\right\rangle\right)$ is a hyperplane of $\operatorname{PG}\left(\bigwedge^{2} V\right)$; more in detail, $\Sigma$ is the kernel of the functional $\beta$ arising from the alternating bilinear form introduced above.

If $\Pi$ is a hyperplane of $\operatorname{PG}\left(\bigwedge^{2} V\right)$ different form $\Sigma$, then $\Pi_{\Sigma}:=\Pi \cap \Sigma$ is a hyperplane of $\Sigma$; clearly, every hyperplane of $\Sigma$ can be obtained by intersecting $\Sigma$ with suitable hyperplanes of $\operatorname{PG}\left(\bigwedge^{2} V\right)$.

So, by Equation (1), $d_{\min }=N-\max _{\Pi}\left|\varepsilon_{2}\left(\Delta_{2}\right) \cap \Pi_{\Sigma}\right|$, where $\Pi$ ranges among all hyperplanes of $\operatorname{PG}\left(\bigwedge^{2} V\right)$ different from $\Sigma$. Regarding $\Pi$ as (the kernel of) a linear functional $\varphi_{\Pi} \in \bigwedge^{2} V^{*}$ we see that the cardinality of $\varepsilon_{2}\left(\Delta_{2}\right) \cap \Pi_{\Sigma}$ is the same as the number of lines of $V$ which are simultaneously totally singular for $\eta$ and totally isotropic for $\varphi_{\Pi}$, now considered as a bilinear alternating form on $V \times V$. Observe that by the correspondence between codewords of $\mathcal{P}_{n, 2}$ and elements of $\bigwedge^{2} V^{*} / \mathcal{N}\left(\varepsilon_{2}\left(\Delta_{2}\right)\right)$ explained in Section 2.1. two functionals $\varphi, \vartheta \in \bigwedge^{2} V^{*}$ induce the same codeword $c \in \mathcal{P}_{n, 2}$ if and only if $\varphi-\vartheta=a \beta$, for some $a \in \mathbb{F}_{q}$.

As $\operatorname{dim}(V)$ is odd, the bilinear form $\varphi_{\Pi}$ is always degenerate; denote by $\operatorname{Rad}\left(\varphi_{\Pi}\right)$ its radical, i.e. $\operatorname{Rad}\left(\varphi_{\Pi}\right):=\left\{x \in V: \varphi_{\Pi}(x, y)=0 \forall y \in V\right\}$. 
We are now ready to prove our main theorem. We proceed in several stages. First of all we consider in Section 3.1 those hyperplanes $\Pi$ corresponding to alternating bilinear forms having radical $\operatorname{Rad}\left(\varphi_{\Pi}\right)$ containing the nucleus $N$ of $\mathcal{Q}$. We prove in Lemma 3.1 that the weight of these forms is always at least $q^{4 n-5}-q^{2 n-3}$, thus showing that they cannot have minimum weight. Then, in Section 3.2 we deal with the class of hyperplanes corresponding to alternating bilinear forms having radical not containing the nucleus $N$ of $\mathcal{Q}$. We show that a necessary condition for the forms to correspond to minimal weight codewords is to have radical of maximum dimension (see Theorem 3.6). Finally, we characterize the codewords of minimum weight.

\subsection{Weight of $\varphi_{\Pi}$ when $N \subseteq \operatorname{Rad}\left(\varphi_{\Pi}\right)$}

Suppose $\Pi$ to be a hyperplane of $\operatorname{PG}\left(\bigwedge^{2} V\right)$ corresponding to a bilinear alternating form $\varphi_{\Pi}$ whose radical $\operatorname{Rad}\left(\varphi_{\Pi}\right)$ contains $N$. For the sake of simplicity, we will write $\varphi$ instead of $\varphi_{\Pi}$.

Denote by $\mathcal{W}$ the non-degenerate symplectic polar space in $V / N$ having as points the lines of $V$ through $N$ and as lines the planes of $V$ through $N$ containing a line of $\mathcal{Q}$. It is immediate to see that $\mathcal{W}$ is defined by the non-degenerate alternating form $\beta^{\text {sp }}$ induced by $\beta$ on $V / N$.

The projection $\iota$ from $V$ to $V / N$ induces an isomorphism of polar spaces from $\mathcal{Q}$ to $\mathcal{W}$. So, $\iota$ naturally induces an isomorphism $\iota$ between the orthogonal line-Grassmannian $\Delta_{2}$ and the symplectic line-Grassmannian $\Delta_{2}^{\mathrm{sp}}$ associated with $\beta^{\mathrm{sp}}$ by $\bar{\imath}(\ell):=\langle\ell, N\rangle / N$ for any $\ell \in \Delta_{2}$. Denote by $\mathcal{P}_{n, 2}^{\mathrm{sp}}$ the symplectic Grassmann code defined by $\beta^{\mathrm{sp}}$ on $V / N$. (See [4] for more information on symplectic Grassmann codes.)

Lemma 3.1. If $N \subseteq \operatorname{Rad}(\varphi)$ then $\operatorname{wt}(\varphi) \geq q^{4 n-5}-q^{2 n-3}$.

Proof. Since $N \subseteq \operatorname{Rad}(\varphi)$, we can consider the (possibly degenerate) symplectic polar space $\mathcal{W}_{\varphi}$ of $V / N$ having as points the lines of $V$ (totally isotropic for $\varphi$ ) through $N$ and as lines the planes of $V$ through $N$ which are totally isotropic for $\varphi$. Denote by $\varphi^{\text {sp }}$ the bilinear alternating form of $V / N$ defining $\mathcal{W}_{\varphi}$. Clearly, $\varphi^{\mathrm{sp}}$ is the form induced by $\varphi$ on $V / N$. Since $N \subseteq \operatorname{Rad}(\varphi)$, given any line $\ell$ of $V$ with $N \notin \ell$ which is totally isotropic for $\varphi$, the plane $\langle N, \ell\rangle$ is also totally isotropic for $\varphi$. So $\bar{l}(\ell)$ is a totally isotropic line for $\varphi^{\mathrm{sp}}$. Conversely, if $\langle\ell, N\rangle$ is a totally isotropic line for $\varphi^{\mathrm{sp}}$, then $\ell$ is totally isotropic for $\varphi$. So, the projection $\iota: V \rightarrow V / N$ induces a bijection between the set of lines of $V$ which are simultaneously totally singular for $\eta$ and totally isotropic for $\varphi$ and the set of lines of $V / N$ which are simultaneously totally isotropic for $\beta^{\text {sp }}$ and totally isotropic for $\varphi^{\mathrm{sp}}$. So $\operatorname{wt}(\varphi)=\operatorname{wt}\left(\varphi^{\mathrm{sp}}\right)$, where $\operatorname{wt}(\varphi)$ is intended as the weight of the codeword induced by $\varphi$ in the orthogonal line-Grassmann code $\mathcal{P}_{n, 2}$, while $\operatorname{wt}\left(\varphi^{\mathrm{sp}}\right)$ corresponds to the weight of a codeword $c_{\varphi^{\mathrm{sp}}}$ in the symplectic line-Grassmann code $\mathcal{P}_{n, 2}^{\mathrm{sp}}$. By the Main Theorem of [4], $\operatorname{wt}\left(\varphi^{\mathrm{sp}}\right) \geq q^{4 n-5}-q^{2 n-3}$. This completes the lemma.

Observe that the isomorphism $\bar{\imath}$ is not an isomorphism between the codes $\mathcal{P}_{n, 2}$ and $\mathcal{P}_{n, 2}^{\mathrm{sp}}$ induced by the projective systems arising from the embedding of the respective polar Grassmannians; in particular, we see that for $q$ even $\mathcal{P}_{n, 2}^{\text {sp }}$ is a proper subcode of $\mathcal{P}_{n, 2}$ with codimension $2 n$.

\subsection{Weight of $\varphi_{\Pi}$ when $N \nsubseteq \operatorname{Rad}\left(\varphi_{\Pi}\right)$}

Suppose that $\Pi$ is a hyperplane of $\operatorname{PG}\left(\bigwedge^{2} V\right)$ corresponding to a bilinear alternating form $\varphi_{\Pi}$ whose radical $\operatorname{Rad}\left(\varphi_{\Pi}\right)$ does not contain $N$. As in Section 3.1, we shall write $\varphi$ instead of $\varphi_{\Pi}$. It is not possible to proceed now as in Section 3.1 since, under the hypothesis $N \nsubseteq \operatorname{Rad}(\varphi)$, the form $\varphi$ does not induce any symplectic polar space in $V / N$, as there are some lines of $V$ through $N$ which are not totally isotropic for $\varphi$. 
We will rely on Equation (2) adapted to the special case $\Omega=\varepsilon_{2}\left(\Delta_{2}\right)$. For the convenience of the reader we write explicitly Equation (2) in this case: for any $\varphi \in \bigwedge^{2} V$,

$$
\operatorname{wt}(\varphi)=\frac{1}{q^{2}-1} \sum_{u \in \mathcal{Q}} \operatorname{wt}\left(\widetilde{\varphi}_{u}\right)
$$

where $\widetilde{\varphi}_{u}:\left(u^{\perp \mathcal{Q}} /\langle u\rangle\right) \rightarrow \mathbb{F}_{q}, \widetilde{\varphi}_{u}(x+\langle u\rangle):=\varphi(u, x)$ with $x \in u^{\perp \mathcal{Q}}$ and $u \in \mathcal{Q}$. Observe that the vector space $u^{\perp \mathcal{Q}} /\langle u\rangle$ is naturally endowed with the quadratic form $\eta_{u}: x+\langle u\rangle \rightarrow \eta(x)$ and $\operatorname{dim} u^{\perp \mathcal{Q}} /\langle u\rangle=2 n-1$. It is well known that the set of all totally singular points for $\eta_{u}$ is a parabolic quadric $\mathcal{Q}_{u} \cong Q(2 n-2, q)$ of rank $n-1$ in $u^{\perp \mathcal{Q}} /\langle u\rangle$. In particular, the possible non-zero weights of $\widetilde{\varphi}_{u}$ correspond to the non-trivial hyperplane sections of $\mathcal{Q}_{u}$. So, the following lemma is straightforward.

Lemma 3.2. Either $\widetilde{\varphi}_{u}=0$ or $\operatorname{wt}\left(\widetilde{\varphi}_{u}\right) \in\left\{q^{2 n-3}-q^{n-2}, q^{2 n-3}, q^{2 n-3}+q^{n-2}\right\}$.

Define

$$
\begin{array}{ll}
\mathfrak{A}^{\prime}:=\left\{u: u \in \mathcal{Q} \text { and } \widetilde{\varphi}_{u} \neq 0\right\}, & A^{\prime}:=\left|\mathfrak{A}^{\prime}\right| ; \\
\mathfrak{B}:=\left\{u: u \in \mathfrak{A}^{\prime} \text { and } \operatorname{wt}\left(\widetilde{\varphi}_{u}\right)=q^{2 n-3}\right\}, & B:=|\mathfrak{B}| ; \\
\mathfrak{C}:=\left\{u: u \in \mathfrak{A}^{\prime} \text { and } \operatorname{wt}\left(\widetilde{\varphi}_{u}\right)=q^{2 n-3}+q^{n-2}\right\}, & C:=|\mathfrak{C}| .
\end{array}
$$

By definition, both $\mathfrak{B}$ and $\mathfrak{C}$ are subsets of $\mathfrak{A}^{\prime}$ and $B, C \geq 0$. Using Equation (4) and Lemma 3.2 we can write

$$
\mathrm{wt}(\varphi)=\frac{q^{2 n-3}-q^{n-2}}{q^{2}-1} A^{\prime}+\frac{q^{n-2}}{q^{2}-1} B+\frac{2 q^{n-2}}{q^{2}-1} C .
$$

For any $u \in V$, write $u^{\perp \varphi}:=\{x \in V: \varphi(u, x)=0\} \subseteq V$.

Lemma 3.3. Let $u \in \mathcal{Q}$. Then $\widetilde{\varphi}_{u}=0 \Leftrightarrow u^{\perp \mathcal{Q}} \subseteq u^{\perp_{\varphi}}$.

Proof. Let $x \in u^{\perp_{\mathcal{Q}}}$ and suppose $u^{\perp_{\mathcal{Q}}} \subseteq u^{\perp_{\varphi}}$. Then $\varphi(u, x)=0$. So, by definition of $\varphi_{u}, \varphi_{u}(x)=0$ for any $x \in u^{\perp \mathcal{Q}}$, whence $\widetilde{\varphi}_{u}=0$. Conversely, if $\widetilde{\varphi}_{u}$ is identically zero, then $\varphi_{u}(x)=\varphi(u, x)=$ $0 \forall x \in u^{\perp \mathcal{Q}} ;$ so $u^{\perp \mathcal{Q}} \subseteq u^{\perp_{\varphi}}$.

Put

$$
S:=\left|\left\{u \in \mathcal{Q}: \widetilde{\varphi}_{u}=0\right\}\right|=q^{2 n}-1-A^{\prime} .
$$

By Lemma 3.3, $S=\left|\left\{u \in \mathcal{Q}: u^{\perp_{\mathcal{Q}}} \subseteq u^{\perp_{\varphi}}\right\}\right|=\left|\left\{u \in \mathcal{Q}: u^{\perp_{\varphi}}=V\right\}\right|+\left|\left\{u \in \mathcal{Q}: u^{\perp_{\mathcal{Q}}}=u^{\perp_{\varphi}}\right\}\right|$.

If we define $A:=q^{2 n-2}-1-S$, then Equation (5) becomes

$$
\operatorname{wt}(\varphi)=q^{4 n-5}-q^{3 n-4}+\frac{q^{n-2}}{q^{2}-1}\left(\left(q^{n-1}-1\right) A+B+2 C\right) .
$$

Furthermore,

$$
\begin{aligned}
A & =q^{2 n-2}-1-\left|\left\{u \in \mathcal{Q}: u^{\perp \mathcal{Q}} \subseteq u^{\perp_{\varphi}}\right\}\right| \\
& =q^{2 n-2}-1-|\{\operatorname{Rad}(\varphi) \cap \mathcal{Q}\}|-\left|\left\{u \in \mathcal{Q}: u^{\perp_{\mathcal{Q}}}=u^{\perp_{\varphi}}\right\}\right| .
\end{aligned}
$$

In particular, as $B, C \geq 0$, if $A>0$, then $\operatorname{wt}(\varphi)>q^{4 n-5}-q^{3 n-4}$.

We shall first consider non-null bilinear forms $\varphi$ whose radical is not maximum and show that they cannot give words of weight $q^{4 n-5}-q^{3 n-4}$. Then we shall study in detail the weights arising from bilinear forms $\varphi$ whose radical has dimension $2 n-1$.

Lemma 3.4. If $N \nsubseteq \operatorname{Rad}(\varphi)$ and $\operatorname{dim}(\operatorname{Rad}(\varphi))<2 n-1$, then $A>0$. If $n \geq 3$, then $A \geq$ $q^{2 n-2}-q^{2 n-3}-q^{2}>0$ 
Proof. Write $\operatorname{dim}(\operatorname{Rad}(\varphi))=2(n-r)+1$ with $1<r \leq n$. By Equation (7), in order to prove $A>0$, we need to provide a suitable upper bound on the cardinality of the set $\left\{u \in \mathcal{Q}: u^{\perp \mathcal{Q}} \subseteq\right.$ $\left.u^{\perp \varphi}\right\}$.

Since $N$ is the nucleus of the quadric $\mathcal{Q}$, we have $N \subseteq u^{\perp \mathcal{Q}}$ for any $u \in \mathcal{Q}$; so, $N \subseteq u^{\perp \varphi}$ for any $u \in \mathcal{Q}$ such that $u^{\perp \mathcal{Q}} \subseteq u^{\perp_{\varphi}}$. Consequently, $u \in N^{\perp_{\varphi}}$ for any $u \in \mathcal{Q}$ such that $u^{\perp \mathcal{Q}} \subseteq u^{\perp_{\varphi}}$. Note that $\Pi_{\varphi}:=N^{\perp_{\varphi}}$ is a (proper) hyperplane of $V$ since $N \nsubseteq \operatorname{Rad}(\varphi)$. Equation (77) can now be rewritten as

$$
A=q^{2 n-2}-1-|\{\operatorname{Rad}(\varphi) \cap \mathcal{Q}\}|-\left|\left\{u \in \mathcal{Q} \cap \Pi_{\varphi}: u^{\perp_{\mathcal{Q}}}=u^{\perp_{\varphi}}\right\}\right| .
$$

Denote by $\bar{V}$ the $2 n$-dimensional vector space spanned by $\bar{B}:=\left(e_{i}\right)_{i=1}^{2 n}$ and for any vector $x \in V$ of coordinates $\left(x_{i}\right)_{i=1}^{2 n+1}$ with respect to the basis $B$, let $\bar{x}$ be the vector of $\bar{V}$ with coordinates $\left(x_{i}\right)_{i=1}^{2 n}$ with respect to $\bar{B}$. The map $\xi: x \rightarrow \bar{x}$ is clearly linear from $V$ to $\bar{V}$. We warn the reader that $\bar{V}$ shall not be regarded as a subspace of $V$. Given any $\bar{x}=\left(x_{i}\right)_{i=1}^{2 n} \in \bar{V}$ there is exactly one vector $x \in \mathcal{Q}$ such that $x=\left(x_{i}\right)_{i=1}^{2 n+1}$; here, $x_{2 n+1}=\left(x_{1} x_{2}+\cdots+x_{2 n-1} x_{2 n}\right)^{1 / 2}$. In particular the restriction of $\xi$ to $\mathcal{Q}$ is a bijection to $\bar{V}$.

Let $S$ be the $(2 n+1) \times(2 n+1)$-antisymmetric matrix representing $\varphi$ with respect to $B$ and consider the alternating bilinear form $\bar{\varphi}: \bar{V} \times \bar{V} \rightarrow \mathbb{F}_{q}$ represented (with respect to $\bar{B}$ ) by the matrix $\bar{S}$ obtained from $S$ by removing its last row and its last column. More explicitly, if

$S:=\left(\begin{array}{ccccc}0 & s_{12} & \ldots & s_{1,2 n} & s_{1,2 n+1} \\ s_{12} & 0 & \ldots & s_{2,2 n} & s_{2,2 n+1} \\ \vdots & \vdots & & \vdots & \vdots \\ s_{1,2 n+1} & s_{2,2 n+1} & \ldots & s_{2 n, 2 n+1} & 0\end{array}\right) \quad$ then $\quad \bar{S}:=\left(\begin{array}{cccc}0 & s_{12} & \ldots & s_{1,2 n} \\ s_{12} & 0 & \ldots & s_{2,2 n} \\ \vdots & & & \vdots \\ s_{1,2 n} & s_{2,2 n} & \ldots & 0\end{array}\right)$.

Analogously, let $M$ be the $(2 n+1) \times(2 n+1)$-antisymmetric matrix representing the bilinear form $\beta$ associated to the quadratic form $\eta$ (see Equation (3)) with respect to $B$ and consider the alternating bilinear form $\bar{\beta}: \bar{V} \times \bar{V} \rightarrow \mathbb{F}_{q}$ represented (with respect to $\bar{B}$ ) by the matrix $\bar{M}$ obtained form $M$ by removing its last row and its last column:

$$
M:=\left(\begin{array}{cccccc}
0 & 1 & \ldots & 0 & 0 & 0 \\
1 & 0 & \ldots & 0 & 0 & 0 \\
\vdots & & \ddots & & & \vdots \\
0 & 0 & \ldots & 0 & 1 & 0 \\
0 & 0 & \ldots & 1 & 0 & 0 \\
0 & 0 & \ldots & 0 & 0 & 0
\end{array}\right) \quad \text { and } \quad \bar{M}:=\left(\begin{array}{ccccc}
0 & 1 & \ldots & 0 & 0 \\
1 & 0 & \ldots & 0 & 0 \\
\vdots & & \ddots & & \vdots \\
0 & 0 & \ldots & 0 & 1 \\
0 & 0 & \ldots & 1 & 0
\end{array}\right) .
$$

Note that $\bar{M}$ is non-singular and $\bar{M}^{-1}=\bar{M}$. Under these assumptions $\Pi_{\varphi}=N^{\perp_{\varphi}}$ has equation

$$
\Pi_{\varphi}: \sum_{i=1}^{2 n} s_{i, 2 n+1} x_{i}=0 .
$$

Claim 1. The following properties hold:

a) $\varphi(x, y)=\bar{\varphi}(\bar{x}, \bar{y}), \forall x, y \in \Pi_{\varphi}$;

b) $\beta(x, y)=\bar{\beta}(\bar{x}, \bar{y}), \forall x, y \in \Pi_{\varphi}$.

Proof. We shall only prove Case 国), as Case $\mathrm{b}$ ) is entirely analogous. 
Let $x=\left(x_{i}\right)_{i=1}^{2 n+1}$ and $y=\left(y_{i}\right)_{1}^{2 n+1}$ be the coordinates of two vectors in $\Pi_{\varphi}$. Then, by Equation (9),

$$
\sum_{i=1}^{2 n} s_{i, 2 n+1} y_{i}=0 \quad \text { and } \quad \sum_{i=1}^{2 n} s_{i, 2 n} x_{i}=0 .
$$

So we have

$$
\begin{aligned}
\varphi(x, y) & =\left(x_{1}, x_{2}, \ldots, x_{2 n+1}\right) S\left(\begin{array}{c}
y_{1} \\
y_{2} \\
\vdots \\
y_{2 n+1}
\end{array}\right)=\sum_{\substack{i, j=1 \\
i<j}}^{2 n+1} s_{i j} x_{j} y_{i}+\sum_{\substack{i, j=1 \\
i<j}}^{2 n+1} s_{i j} x_{i} y_{j}= \\
& =\sum_{\substack{i, j=1 \\
i<j}}^{2 n} s_{i j} x_{j} y_{i}+\sum_{\substack{i, j=1 \\
i<j}}^{2 n} s_{i j} x_{i} y_{j}+x_{2 n+1} \sum_{i=1}^{2 n} s_{i, 2 n+1} y_{i}+y_{2 n+1} \sum_{i=1}^{2 n} s_{i, 2 n} x_{i}= \\
& =\left(x_{1}, x_{2}, \ldots, x_{2 n}\right) \bar{S}\left(\begin{array}{c}
y_{1} \\
y_{2} \\
\vdots \\
y_{2 n}
\end{array}\right)+x_{2 n+1} \sum_{i=1}^{2 n} s_{i, 2 n+1} y_{i}+y_{2 n+1} \sum_{i=1}^{2 n} s_{i, 2 n} x_{i}= \\
& =\bar{x}^{T} \bar{S} \bar{y}=\bar{\varphi}(\bar{x}, \bar{y}) .
\end{aligned}
$$

The condition $u^{\perp \mathcal{Q}}=u^{\perp \varphi}$ with $u \notin \operatorname{Rad}(\varphi)$ holds if and only if the systems of equations $x^{t} S u$ and $x^{t} M u$, where $x=\left(x_{i}\right)_{i=1}^{2 n+1}$ are equivalent. This means that there exists an element $\lambda \in \mathbb{F}_{q} \backslash\{0\}$ such that $S u=\lambda M u$. Note that for $\lambda=0$ we have vectors $u$ in $\operatorname{Rad}(\varphi)$ and the inclusion $u^{\perp \mathcal{Q}} \subseteq u^{\perp \varphi}$ is proper. The set

$$
U:=\left\{u \in \Pi_{\varphi} \cap \mathcal{Q}: \exists \lambda \neq 0 \text { such that } v^{T} S u=\lambda v^{T} M u, \quad \forall v \in V\right\}
$$

is clearly a proper subset of

$$
\widetilde{U}:=\left\{u \in \Pi_{\varphi} \cap \mathcal{Q}: \exists \lambda \neq 0 \text { such that } v^{T} S u=\lambda v^{T} M u, \quad \forall v \in \Pi_{\varphi}\right\} .
$$

By Claim 1 we have

$$
\begin{aligned}
\widetilde{U} & =\left\{u \in \Pi_{\varphi} \cap \mathcal{Q}: \exists \lambda \neq 0 \text { such that } \bar{v}^{T} \bar{S} \bar{u}=\lambda \bar{v}^{T} \bar{M} \bar{u}, \quad \forall v \in \Pi_{\varphi}\right\}= \\
& =\left\{u \in \Pi_{\varphi} \cap \mathcal{Q}: \exists \lambda \neq 0 \text { such that } \bar{S} \bar{u}=\lambda \bar{M} \bar{u}\right\}= \\
& =\left\{u \in \Pi_{\varphi} \cap \mathcal{Q}: \bar{u} \text { is an eigenvector of non-zero eigenvalue for } \overline{M S}\right\} .
\end{aligned}
$$

Claim 2. The number of eigenvectors of $\overline{M S}$ of non-zero eigenvalue is at most $q^{2 r-2}$.

Proof. Let $V_{0}:=\operatorname{ker}(\overline{M S})$ be the eigenspace of eigenvalue 0 of $\overline{M S}$.

To prove Claim 2 we shall first show that $\operatorname{dim}\left(V_{0}\right)=\operatorname{dim}(\operatorname{Rad}(\varphi))+1$. As $\bar{M}$ is nonsingular, $V_{0}=\operatorname{ker}(\bar{S})$. Furthermore, since $\bar{S}$ is a $(2 n \times 2 n)$-minor of $S$, we have $\operatorname{rank}(S)-2 \leq$ $\operatorname{rank}(\bar{S}) \leq \operatorname{rank}(S)$. In particular, $\operatorname{dim}(\operatorname{Rad}(\varphi))-1 \leq \operatorname{dim} V_{0} \leq \operatorname{dim}(\operatorname{Rad}(\varphi))+1$. Define $\overline{\operatorname{Rad}}(\varphi):=\{\bar{x}: x \in \operatorname{Rad}(\varphi)\}$. We claim that $\overline{\operatorname{Rad}}(\varphi)$ is a proper subspace of $V_{0}$. Indeed, let $\bar{w} \in \overline{\operatorname{Rad}}(\varphi)$. As $\operatorname{Rad}(\varphi) \subset \Pi_{\varphi}$ and $w \in \operatorname{Rad}(\varphi)$ we have, by Claim 1, that $\bar{\varphi}(\bar{w}, \bar{x})=0=$ $\varphi(w, x)$ for any $x \in \Pi_{\varphi}$. This implies $\bar{S} \bar{w}=0$; so $\bar{w} \in V_{0}$. Furthermore, $\operatorname{dim}(\overline{\operatorname{Rad}}(\varphi))=$ $\operatorname{dim}(\operatorname{Rad}(\varphi))$. Indeed, let $\left(b_{1}, \ldots, b_{2(n-r)+1}\right)$ be a basis of $\operatorname{Rad}(\varphi)$ then $\left(\bar{b}_{1}, \ldots, \bar{b}_{2(n-r)+1}\right)$ is clearly a generating set for $\overline{\operatorname{Rad}}(\varphi)$. If the latter vectors were to be linearly dependent, then there 
would be $\alpha_{1}, \ldots, \alpha_{2(n-r)+1}$, not all zero, such that $\alpha_{1} \bar{b}_{1}+\cdots+\alpha_{2(n-r)+1} \bar{b}_{2(n-r)+1}=0$. Then $v:=$ $\alpha_{1} b_{1}+\cdots+\alpha_{2(n-r)+1} b_{2(n-r)+1} \neq 0$ and $\bar{v}=0$. This means $v=\gamma e_{2 n+1} \in \operatorname{Rad}(\varphi)$ for some $\gamma \neq 0$, a contradiction as $N \nsubseteq \mathbb{R a d}(\varphi)$. So, $\operatorname{dim}\left(V_{0}\right) \geq \operatorname{dim}(\overline{\operatorname{Rad}}(\varphi))=\operatorname{dim}(\operatorname{Rad}(\varphi))=2(n-r)+1$. Since $S$ is an antisymmetric matrix of odd order, $\operatorname{dim}(\overline{\operatorname{Rad}}(\varphi))=\operatorname{dim}(\operatorname{Rad}(\varphi))=\operatorname{dim}(\operatorname{ker}(S))$ is odd. On the other hand $\bar{S}$ is, by construction, an antisymmetric matrix of even order, so $\operatorname{dim}\left(V_{0}\right)=\operatorname{dim}(\operatorname{ker}(\bar{S}))$ is even; hence $\operatorname{dim}\left(V_{0}\right) \neq \operatorname{dim}(\operatorname{Rad}(\varphi))=2(n-r)+1$. It follows that

$$
\operatorname{dim}\left(V_{0}\right)=2 n-2 r+2=\operatorname{dim}(\operatorname{Rad}(\varphi))+1 .
$$

Suppose now that there are $t \geq 0$ eigenspaces $V_{\lambda_{1}}, \ldots, V_{\lambda_{t}}$ of $\overline{M S}$ of non-zero eigenvalues $\lambda_{1}, \ldots, \lambda_{t}$ and let $d_{i}:=\operatorname{dim}\left(V_{\lambda_{i}}\right) \geq 1$. Note that if $t=0$ then we immediately have $A>0$. Suppose also $d_{1} \leq d_{2} \leq \cdots \leq d_{t}$. Then,

$$
\sum_{i=1}^{t} d_{i}+\operatorname{dim}\left(V_{0}\right) \leq \operatorname{dim}(\bar{V}) .
$$

By Equation (13), we have

$$
\sum_{i=1}^{t} d_{i} \leq 2 n-\operatorname{dim}\left(V_{0}\right) \leq 2 r-2
$$

so, by the properties of the exponential function,

$$
\sum_{i=1}^{t}\left(\left|V_{\lambda_{i}}\right|-1\right)=\sum_{i=1}^{t}\left(q^{d_{i}}-1\right) \leq q^{\sum_{i=1}^{t} d_{i}}-t \leq q^{2 r-2} .
$$

Suppose $3 \leq \operatorname{dim}(\operatorname{Rad}(\varphi)) \leq 2 n-3$. By Equation (7),

$$
A=q^{2 n-2}-1-|\{\operatorname{Rad}(\varphi) \cap \mathcal{Q}\}|-\left|\left\{u \in \mathcal{Q}: u^{\perp \mathcal{Q}}=u^{\perp \varphi}\right\}\right| ;
$$

using Equations (8), (10), (11) and (12),

$$
A \geq q^{2 n-2}-1-(|\operatorname{Rad}(\varphi)|-1)-|U| \geq q^{2 n-2}-q^{2 n-2 r+1}-|\widetilde{U}| .
$$

By Claim 2, $|\widetilde{U}| \leq q^{2 r-2}$; hence,

$$
A \geq q^{2 n-2}-q^{2 n-2 r+1}-q^{2 r-2} .
$$

Under the assumption $3 \leq \operatorname{dim}(\operatorname{Rad}(\varphi)) \leq 2 n-3$, we have $2 \leq r \leq n-1$. So, $2 r-2 \leq 2 n-4$ and $2 n+1-2 r \leq 2 n-3$. By taking these two inequalities into account in Equation (15) we get

$$
A \geq q^{2 n-2}-q^{2 n-2 r+1}-q^{2 r-2}
$$

Observe that the function $f(r):=q^{2 n-2 r+1}+q^{2 r-2}$, regarded as defined over the reals, has derivative $\frac{\partial f}{\partial r}=2 \log (q)\left(q^{2 r-2}-q^{2 n-2 r+1}\right)$. In particular $f(r)$ is decreasing for $2 \leq r<\frac{2 n+3}{2}$. So it attains its maximum for $r=2$ and

$$
A \geq q^{2 n-2}-q^{2 n-3}-q^{2}>0 .
$$

Note that if $r=n$ the last inequality does not hold. This completes the proof for $3 \leq$ $\operatorname{dim}(\operatorname{Rad}(\varphi)) \leq 2 n-3$. 
Suppose $\operatorname{dim}(\operatorname{Rad}(\varphi))=1$. This is equivalent to say $r=n$. In this case, by Equation (13), $\operatorname{dim} V_{0}=\operatorname{dim}(\operatorname{ker}(\bar{S}))=2$. By Equation (14), the maximum dimension of an eigenspace of $\overline{M S}$ is $2 n-2$.

Define

$$
\bar{\Pi}_{\varphi}:=\left\{\bar{x}: x \in \mathcal{Q} \cap \Pi_{\varphi}\right\} .
$$

Then, $\bar{\Pi}_{\varphi}$ is the hyperplane of $\bar{V}$ of equation $\sum_{i=1}^{2 n} s_{i, 2 n+1} x_{i}=0$ and the map $\xi: x \rightarrow \bar{x}$ is a bijection between the points of $\mathcal{Q} \cap \Pi_{\varphi}$ and those of $\bar{\Pi}_{\varphi}$.

Claim 3.

$$
|\widetilde{U}|=\sum_{\lambda \neq 0}\left|\bar{\Pi}_{\varphi} \cap V_{\lambda}\right|
$$

Proof. For $\lambda$ a non-zero eigenvalue of $\overline{M S}$, define $\widetilde{U}_{\lambda}:=\left\{u \in \Pi_{\varphi} \cap \mathcal{Q}: \overline{M S} \bar{u}=\lambda \bar{u}\right\}$. By the above considerations, $\left|\widetilde{U}_{\lambda}\right|=\left|\bar{\Pi}_{\varphi} \cap V_{\lambda}\right|$. Furthermore, $\widetilde{U}_{\lambda} \cap \widetilde{U}_{\mu}=\emptyset$ for $\lambda \neq \mu$ and, by (12), $\widetilde{U}=\bigcup_{\lambda \neq 0} \widetilde{U}_{\lambda}$. This proves the claim.

Claim 4. Assume that there are $t>0$ distinct eigenspaces $V_{\lambda_{i}}$ for $\overline{M S}$ of non-zero eigenvalue. Then,

$$
\sum_{i=1}^{t}\left|\bar{\Pi}_{\varphi} \cap V_{\lambda_{i}}\right| \leq\left|\bar{\Pi}_{\varphi} \cap Z\right|
$$

where $Z=\oplus_{i=1}^{t} V_{\lambda_{i}}$ and $\operatorname{dim}(Z) \leq 2 n-2$.

Proof. Suppose $t \geq 2$. Take two eigenspaces $V_{\lambda_{1}}$ and $V_{\lambda_{2}}$ of $\overline{M S}$ with dimension respectively $d_{1}, d_{2} \leq 2 n-2$ and define $Z:=V_{\lambda_{1}} \oplus V_{\lambda_{2}}$. As $V_{\lambda_{1}} \cup V_{\lambda_{2}} \leq Z$, we have $\left|\bar{\Pi}_{\varphi} \cap V_{\lambda_{1}}\right|+\left|\bar{\Pi}_{\varphi} \cap V_{\lambda_{2}}\right|=$ $\left|\bar{\Pi}_{\varphi} \cap\left(V_{\lambda_{1}} \cup V_{\lambda_{2}}\right)\right| \leq\left|\bar{\Pi}_{\varphi} \cap Z\right|$. So

$$
\sum_{i=1}^{t}\left|V_{\lambda_{i}} \cap \bar{\Pi}_{\varphi}\right| \leq\left|Z \cap \bar{\Pi}_{\varphi}\right|+\sum_{i=3}^{t}\left|V_{\lambda_{i}} \cap \bar{\Pi}_{\varphi}\right| .
$$

Iterating this procedure $t-1$ times we get $\sum_{i=1}^{t}\left|V_{\lambda_{i}} \cap \bar{\Pi}_{\varphi}\right| \leq\left|Z^{\prime} \cap \bar{\Pi}_{\varphi}\right|$ where $Z^{\prime}:=\oplus_{i=1}^{t} V_{\lambda_{i}}$. As $\sum d_{i} \leq 2 n-2$, we have the claim.

Using Claim 4, we see that a matrix $\overline{M S}$ having the maximum number of eigenvectors (of nonnull eigenvalues) can be taken so that it admits exactly one eigenspace $V_{\lambda}$ with $\operatorname{dim} V_{\lambda}=2 n-2$. We shall assume this to be the case in the remainder of the section. So, by Equation (12) and Claim 3 ,

$$
A \geq q^{2 n-2}-1-(q-1)-\sum_{i=1}^{t}\left|\bar{\Pi}_{\varphi} \cap V_{\lambda_{i}}\right| \geq q^{2 n-2}-q-\left|V_{\lambda} \cap \bar{\Pi}_{\varphi}\right| .
$$

Recall that $\bar{\Pi}_{\varphi}$ is a hyperplane of $\bar{V}$; so $\left|V_{\lambda} \cap \bar{\Pi}_{\varphi}\right|$ can assume only two values depending on whether $\bar{\Pi}_{\varphi}$ intersects $V_{\lambda}$ in a hyperplane or $\bar{\Pi}_{\varphi}$ properly contains $V_{\lambda}$. In the former case, $\operatorname{dim}\left(V_{\lambda} \cap \bar{\Pi}_{\varphi}\right)=2 n-3$; hence $\left|V_{\lambda} \cap \bar{\Pi}_{\varphi}\right|=q^{2 n-3}$ and Equation (16) gives $A>0$, proving the lemma.

In the latter case, Equation (16) is not sufficient, as it gives $A \geq-q$. To rule out this possibility we need a more accurate lower bound for $A$. To this aim, consider Equation (77) under the assumption $V_{\lambda} \subseteq \bar{\Pi}_{\varphi}$. We have

$$
A \geq q^{2 n-2}-1-(q-1)-\left|\left\{u \in \mathcal{Q} \cap \Pi_{\varphi}: u^{\perp \mathcal{Q}}=u^{\perp \varphi}\right\}\right| .
$$


Also,

$\left|\left\{u \in \mathcal{Q} \cap \Pi_{\varphi}: u^{\perp \mathcal{Q}}=u^{\perp \varphi}\right\}\right|=\left|\left\{u \in \Pi_{\varphi} \cap \mathcal{Q}: x^{t} S u=\lambda x^{t} M u, \forall x \in V\right\}\right|=$

$=\left|\left\{u \in \mathcal{Q} \cap \Pi_{\varphi}: x^{t}(S-\lambda M) u=0, \forall x \in V\right\}\right| \leq\left|\left\{u \in \Pi_{\varphi}: u \in \operatorname{ker}(S-\lambda M)\right\}\right|$.

Observe that to any vector in $\bar{y} \in \operatorname{ker}(\bar{S}-\lambda \bar{M})$ there correspond at most one vector $y \in$ $\operatorname{ker}(S-\lambda M)$ because if $y_{1}, y_{2} \in \operatorname{ker}(S-\lambda M), y_{1} \neq y_{2}$ and $\bar{y}_{1}=\bar{y}_{2} \in \operatorname{ker}(\bar{S}-\lambda \bar{M})$, then $\left\langle y_{1}-y_{2}\right\rangle=\left\langle e_{2 n+1}\right\rangle=N$ and $e_{2 n+1} \in \operatorname{ker}(S-\lambda M)$. As $M e_{2 n+1}=0$, this implies $x^{T} S e_{2 n+1}=$ $0, \forall x \in V$; hence, $\left\langle e_{2 n+1}\right\rangle=N \subseteq \operatorname{Rad}(\varphi)$, against our hypothesis.

So, $\operatorname{dim}(\operatorname{ker}(\bar{S}-\lambda \bar{M}))-1 \leq \operatorname{dim}(\operatorname{ker}(S-\lambda M)) \leq \operatorname{dim}(\operatorname{ker}(\bar{S}-\lambda \bar{M}))$. By construction, $\operatorname{dim}(\operatorname{ker}(\bar{S}-\lambda \bar{M}))=\operatorname{dim}\left(V_{\lambda}\right) \leq 2 n-2$; furthermore, $\operatorname{dim}(\operatorname{ker}(S-\lambda M))$ is odd because $S-\lambda M$ is a $(2 n+1) \times(2 n+1)$-antisymmetric matrix. $\operatorname{So}, \operatorname{dim}(\operatorname{ker}(S-\lambda M))=2 n-3$.

As $(n, q) \neq(2,2)$, by Equations (77) and (17) we have

$$
A \geq q^{2 n-2}-1-(q-1)-|\{u \in V: u \in \operatorname{ker}(S-\lambda M)\}| \geq q^{2 n-2}-q^{2 n-3}-q>0 .
$$

This proves the lemma.

Combining Equation (6), Lemma 3.1 and Lemma 3.4, we have the following.

Corollary 3.5. If $N \subseteq \operatorname{Rad}(\varphi)$ or $N \nsubseteq \operatorname{Rad}(\varphi)$ and $\operatorname{dim}(\operatorname{Rad}(\varphi))<2 n-1$, then $\operatorname{wt}(\varphi)>$ $q^{4 n-5}-q^{3 n-4}$.

There remains to consider the class of alternating bilinear forms having radical of maximum dimension not containing the nucleus $N$.

Lemma 3.6. If $N \nsubseteq \operatorname{Rad}(\varphi)$ and $\operatorname{dim}(\operatorname{Rad}(\varphi))=2 n-1$, then $\operatorname{wt}(\varphi) \geq q^{4 n-5}-q^{3 n-4}$. If $\operatorname{dim} \operatorname{Rad}(\varphi)=2 n-1$ and $\operatorname{Rad}(\varphi) \cap \mathcal{Q}$ is a cone of vertex a point $P$ projecting a hyperbolic quadric $Q^{+}(2 n-3, q)$, then $\operatorname{wt}(\varphi)=q^{4 n-5}-q^{3 n-4}$.

Proof. As $\operatorname{dim} \operatorname{Rad}(\varphi)=2 n-1$, a line $\ell$ of $\mathcal{Q}$ is totally isotropic for $\varphi$ if and only if $\ell \cap \operatorname{Rad}(\varphi) \neq$ $\{0\}$. To determine the weight $\operatorname{wt}(\varphi)$ of $\varphi$ we just need to determine the number of totally singular lines of $\mathcal{Q}$ with non-trivial intersection with $\operatorname{Rad}(\varphi)$.

Let $P \in \mathcal{Q} \cap \operatorname{Rad}(\varphi)$, then all lines through $P$ meet $\operatorname{Rad}(\varphi)$ non-trivially. There are exactly $\left(q^{2 n-2}-1\right) /(q-1)$ such lines. Each line $\ell$ contained in $\mathcal{Q} \cap \operatorname{Rad}(\varphi)$ ends up being counted $(q+1)$ times; so we need to determine the number

$$
|(\mathcal{Q} \cap \operatorname{Rad}(\varphi))| \frac{q^{2 n-2}-1}{q-1}-q \mid\{\text { totally singular lines contained in } \operatorname{Rad}(\varphi)\} \mid .
$$

Denote the number of totally singular lines contained in $\operatorname{Rad}(\varphi)$ by $\sigma(\mathcal{Q} \cap \operatorname{Rad}(\varphi))$.

There are four types of sections obtained by intersecting a parabolic quadric $\mathcal{Q}$ with a space $\Pi_{a} \cap \Pi_{b}$ of codimension 2; indeed

1. if $\Pi_{a} \cap \mathcal{Q}$ is an elliptic quadric $Q^{-}(2 n-1, q)$, then $\Pi_{a} \cap \Pi_{b} \cap \mathcal{Q}$ is either a parabolic quadric $Q(2 n-2, q)$ or a cone over an elliptic quadric $Q^{-}(2 n-3, q)$.

2. if $\Pi_{a} \cap \mathcal{Q}$ is a hyperbolic quadric $Q^{+}(2 n-1, q)$, then $\Pi_{a} \cap \Pi_{b} \cap \mathcal{Q}$ is either a parabolic quadric $Q(2 n-2, q)$ or a cone over a hyperbolic quadric $Q^{+}(2 n-3, q)$.

3. if $\Pi_{a}$ is tangent to $\mathcal{Q}$, then $\Pi_{a} \cap \mathcal{Q}$ is a cone over a parabolic quadric $Q(2 n-2, q)$. The possible intersections of $\Pi_{a} \cap \mathcal{Q}$ with $\Pi_{b}$ are now:

(a) a parabolic quadric $Q(2 n-2, q)$ (if $\Pi_{b}$ does not pass through the vertex of $\Pi_{a} \cap \mathcal{Q}$ ); 
(b) a quadric with vertex a line and basis a parabolic quadric $Q(2 n-4, q)$;

(c) a cone over a hyperbolic quadric $Q^{+}(2 n-3, q)$;

(d) a cone over an elliptic quadric $Q^{-}(2 n-3, q)$.

So $\mathcal{Q} \cap \operatorname{Rad}(\varphi)$ is either:

a) a parabolic quadric $Q(2 n-2, q)$; then,

$$
|\mathcal{Q} \cap \operatorname{Rad}(\varphi)|=\frac{q^{2 n-2}-1}{q-1}, \quad \sigma(\mathcal{Q} \cap \operatorname{Rad}(\varphi))=\frac{\left(q^{2 n-4}-1\right)\left(q^{2 n-2}-1\right)}{\left(q^{2}-1\right)(q-1)} ;
$$

then the weight is

$$
\operatorname{wt}(\varphi)=q^{4 n-5}-q^{2 n-3} ;
$$

b) a cone of vertex a point $P$ over a hyperbolic quadric $Q^{+}(2 n-3, q)$; then,

$$
\begin{gathered}
|\mathcal{Q} \cap \operatorname{Rad}(\varphi)|=q \frac{\left(q^{n-1}-1\right)\left(q^{n-2}+1\right)}{q-1}+1 ; \\
\sigma(\mathcal{Q} \cap \operatorname{Rad}(\varphi))=\frac{\left(q^{n-1}-1\right)\left(q^{n-2}+1\right)}{q-1}+q^{2} \frac{\left(q^{2 n-4}-1\right)\left(q^{n-1}-1\right)\left(q^{n-3}+1\right)}{\left(q^{2}-1\right)(q-1)} ;
\end{gathered}
$$

then the weight is

$$
\operatorname{wt}(\varphi)=q^{4 n-5}-q^{3 n-4} ;
$$

c) a cone of vertex a point $P$ over an elliptic quadric $Q^{-}(2 n-3, q)$; then,

$$
\begin{gathered}
|\mathcal{Q} \cap \operatorname{Rad}(\varphi)|=q \frac{\left(q^{n-1}+1\right)\left(q^{n-2}-1\right)}{q-1}+1 ; \\
\sigma(\mathcal{Q} \cap \operatorname{Rad}(\varphi))=\frac{\left(q^{n-1}+1\right)\left(q^{n-2}-1\right)}{q-1}+q^{2} \frac{\left(q^{2 n-4}-1\right)\left(q^{n-1}+1\right)\left(q^{n-3}-1\right)}{\left(q^{2}-1\right)(q-1)} ;
\end{gathered}
$$

then the weight is

$$
\operatorname{wt}(\varphi)=q^{4 n-5}+q^{3 n-4} ;
$$

d) a singular quadric with vertex a line $\ell$ and basis a parabolic quadric $Q(2 n-4, q)$; then

$$
\begin{gathered}
|\mathcal{Q} \cap \operatorname{Rad}(\varphi)|=q^{2} \frac{q^{2 n-4}-1}{q+1}+(q+1)=\frac{q^{2 n-2}-1}{q-1} ; \\
\sigma(\mathcal{Q} \cap \operatorname{Rad}(\varphi))=1+q \frac{q^{2 n-4}-1}{q-1}+q^{2}\left(q^{2} \frac{\left(q^{2 n-6}-1\right)\left(q^{2 n-4}-1\right)}{\left(q^{2}-1\right)(q-1)}+\frac{\left(q^{2 n-4}-1\right)}{q-1}\right) ;
\end{gathered}
$$

then the weight is

$$
\operatorname{wt}(\varphi)=q^{4 n-5} .
$$

Theorem 3.7. If $N \nsubseteq \operatorname{Rad}(\varphi)$ then $\operatorname{wt}(\varphi) \geq q^{4 n-5}-q^{3 n-4}$. Moreover, if $N \nsubseteq \operatorname{Rad}(\varphi)$ and $\operatorname{dim}(\operatorname{Rad}(\varphi))=2 n-1$ there exist codewords of weight $q^{4 n-5}-q^{3 n-4}$.

Proof. The theorem follows from Corollary 3.5 and Lemma 3.6.

Combining the result for $(q, n)=(2,2)$, Theorem 3.7 and Lemma 3.6 we have the following. 
Corollary 3.8. All minimum weight codewords of $\mathcal{P}_{n, 2}$ for $q$ even are projectively equivalent.

Corollary 3.9. For $n \neq 3$, the second smallest distance of the code is $q^{4 n-5}-q^{2 n-3}$.

Proof. For $n=2$, using the argument of [1, §2.3.2], the full spectrum of the code can be determined, and the second smallest weight is $q^{3}-q$. For $n=3$ For $n \geq 4$, by Lemma 3.4, when $N \nsubseteq \operatorname{Rad}(\varphi)$ we have $A \geq q^{2 n-2}-q^{2 n-3}-q^{2}$. Plugging this in (6), we immediately obtain $\operatorname{wt}(\varphi)>q^{4 n-5}-q^{2 n-3}$ for $n \geq 4$.

Our Main Theorem follows from Corollary 3.5. Theorem 3.7 Corollary 3.8 and Corollary 3.9.

Remark 3.10. Using the estimates appearing in the proof of [3, Theorem 3.7] in [3, Equation (9)], a straightforward computation shows that the second smallest distance of a line polar Grassmann code of orthogonal type is $q^{4 n-5}-q^{2 n-3}$ for $n>3$ also when $q$ is odd.

We conjecture that also for $n=3$ the second smallest distance of the code is $q^{7}-q^{3}$ (both for $q$ even and $q$ odd).

\section{Acknowledgements}

Both authors are affiliated with GNSAGA of INdAM (Italy) whose support they acknowledge. We thank the anonymous referee of the paper for having pointed out the value of the second smallest distance of the code.

\section{References}

[1] I. Cardinali, L. Giuzzi, Codes and Caps from Orthogonal Grassmannians, Finite Fields Appl. 24 (2013), 148-169.

[2] I. Cardinali, L. Giuzzi, Enumerative coding for Line Polar Grassmannians with applications to codes, Finite Fields Appl. 46 (2017), 107-138.

[3] I. Cardinali, L. Giuzzi, K. Kaipa, A. Pasini, Line Polar Grassmann Codes of Orthogonal Type, J. Pure Applied Algebra. 220 (2016), 1924-1934.

[4] I. Cardinali, L. Giuzzi, Minimum distance of Symplectic Grassmann codes, Linear Algebra Appl. 488 (2016), 124-134.

[5] I. Cardinali, L. Giuzzi, Line Hermitian Grassmann Codes and their Parameters, preprint (arXiv:1706.10255).

[6] I. Cardinali, A. Pasini, Grassmann and Weyl embeddings of orthogonal Grassmannians, J. Algebr. Combin. 38 (2013), 863-888.

[7] I. Cardinali, A. Pasini, Embeddings of line-Grassmannians of polar spaces in Grassmann varieties. Groups of exceptional type, Coxeter groups and related geometries, Springer Proc. Math. Stat., Springer, New Delhi, 82 (2014), 75-109.

[8] S.R. Ghorpade, G. Lachaud, Higher weights of Grassmann Codes, in "Coding theory, cryptography and related areas (Guanajuato, 1998)", Springer-Verlag (2000), 122-131. 
[9] S.R. Ghorpade, A.R. Patil, H.K. Pillai, Decomposable subspaces, linear sections of Grassmann varieties, and higher weights of Grassmann codes, Finite. Fields Appl. 15 (2009), 54-68.

[10] D. Yu. Nogin, Codes associated to Grassmannians, in "Arithmetic, geometry and coding theory (Luminy, 1993)", de Gruyter (1996), 145-154.

[11] C.T. Ryan, An application of Grassmannian varieties to coding theory, Congr. Numer. 57 (1987), 257-271.

[12] C.T. Ryan, Projective codes based on Grassmann varieties, Congr. Numer. 57 (1987), 273279 .

[13] M.A. Tsfasman, S.G. Vlădut, D.Yu. Nogin, Algebraic geometric codes: basic notions, Mathematical Surveys and Monographs 139, American Mathematical Society (2007).

[14] V.K. Wei, Generalized Hamming weights for linear codes, IEEE Trans. Inform. Theory 37 (1991), 1412-1418.

Authors' addresses:

Ilaria Cardinali

Department of Information Engineering and

Mathematics

University of Siena

Via Roma 56, I-53100, Siena, Italy

ilaria.cardinali@unisi.it
Luca Giuzzi

D.I.C.A.T.A.M.

Section of Mathematics

Università di Brescia

Via Branze 43, I-25123, Brescia, Italy luca.giuzzi@unibs.it 